\title{
Clinical value of plasma B-type natriuretic peptide assay in pediatric pneumonia accompanied by heart failure
}

\author{
DAN HU $^{1 *}$, YANG LIU $^{2 *}$, HUIXIAN TAO ${ }^{1}$ and JINPING GAO ${ }^{1}$ \\ ${ }^{1}$ Department of Pediatrics, The First People's Hospital of Zhangjiagang, Zhangjiagang, Jiangsu 215600; \\ ${ }^{2}$ Department of Pediatrics, The Second Affiliated Hospital of Nanchang University, Nanchang, Jiangxi 330006, P.R. China
}

Received August 28, 2014; Accepted June 30, 2015

DOI: $10.3892 / \mathrm{etm} .2015 .2781$

\begin{abstract}
Previous studies have shown that B-type natriuretic peptide (BNP) is useful in differentiating cardiac from pulmonary causes of dyspnea in adults. To date, international guidelines have recommended measurements of circulating BNP as a biomarker for diagnostic and prognostic purposes, as well as therapeutic monitoring, in adults with cardiac diseases, particularly those suffering from acute and chronic heart failure (HF). The aim of the present study was to investigate the differential diagnostic and therapeutic analysis of BNP levels assayed in pediatric pneumonia accompanied by HF. The clinical data of 80 patients with pneumonia, aged 1-3 years, were analyzed. The patients were divided into two groups: Simple pneumonia (46 cases) and pneumonia accompanied by HF (34 cases). All patients underwent two plasma BNP assays: The first one upon admission to the hospital and the second one prior to discharge. The plasma BNP levels of 20 healthy children were used as the negative control. Plasma BNP levels were measured using the Triage ${ }^{\circledR}$ BNP automated immunoassay systems and reagents. Statistical analysis showed that the plasma BNP levels of the patients upon admission were higher in the pneumonia accompanied by HF group compared with those in the simple pneumonia group $(750 \pm 120$ vs. $135 \pm 50 \mathrm{pg} / \mathrm{ml} ; \mathrm{P}<0.05)$. In addition, in the pneumonia accompanied by HF group, the plasma BNP levels of the patients were higher upon admission to the hospital than they were prior to discharge $(750 \pm 120$ vs. $115 \pm 45 \mathrm{pg} / \mathrm{ml} ; \mathrm{P}<0.05)$; therefore, plasma BNP may comprise a sensitive diagnostic and therapeutic evaluative marker for pediatric patients with pneumonia accompanied by HF. This finding could prove invaluable in the clinical diagnosis and treatment of the disease.
\end{abstract}

Correspondence to: Dr Jinping Gao, Department of Pediatrics, The First People's Hospital of Zhangjiagang, 68 Jiyang West Road, Zhangjiagang, Jiangsu 215600, P.R. China

E-mail: jinpinggaomd@163.com

*Contributed equally

Key words: pediatrics, heart failure, pneumonia, brain natriuretic peptide

\section{Introduction}

Cardiac natriuretic peptides (NPs), such as the atrial NP (ANP) and B-type natriuretic peptide (BNP), as well as their associated peptides, comprise a complex family of peptide hormones, which are produced and secreted by the human heart $(1,2)$. The physiological role of BNP is to facilitate the adaptation of the myocardium to strain or stress imposed by a volume and/or pressure load. BNP has emerged as a promising marker for the diagnosis of heart failure (HF), as well as the determination of prognosis and monitoring of treatment effects in adult patients with the disease $(3,4)$.

Prognosis permits clinicians to separate patients with HF into subgroups based on likely health outcomes. This separation encourages effective targeting of therapies to subgroups that are most likely to require and benefit from treatment, while minimizing risks to the other subgroups, such as adverse effects. The Canadian National Institute for Health and Clinical Excellence (NICE) guidelines for adult chronic HF note that higher BNP and N-terminal proBNP (NT-proBNP) levels are associated with poorer prognosis in HF. For adults the NICE guidelines recommend transthoracic Doppler 2D echocardiography and specialist assessment for persons with suspected $\mathrm{HF}$, and $\mathrm{BNP} \geq 100 \mathrm{pg} / \mathrm{ml}$ or NT-proBNP $\geq 400 \mathrm{pg} / \mathrm{ml}$. The 2013 Canadian guidelines recommend using BNP and NT-proBNP to obtain prognostic information on patients with HF (5). In addition, the European HF guidelines contain a table of prognostic factors for adults, which includes both peptides (6).

$\mathrm{HF}$ is a condition in which the heart cannot, at a normal filling pressure, pump blood at a rate proportionate to the demands of the metabolizing tissues (5,7-10). The diagnosis of HF remains a clinical challenge in all settings, particularly with regard to pediatric patients. Pneumonia is a frequently encountered disease in children, and, in severe cases, it may influence their cardiovascular function or even cause HF. It is usually an extremely difficult task to determine whether severe pneumonia is accompanied by HF, mostly due to limited communication between the pediatrician and the child, but also due to the fact that the symptoms often manifest more severely than would be expected from the underlying condition (2). For that reason, the present study focused on investigating the differential diagnostic and therapeutic analysis of the plasma BNP assay levels in pediatric pneumonia accompanied by HF. 


\section{Materials and methods}

Patients. The present study was approved by the Ethics Committee of The First People's Hospital of Zhangjiagang (Zhangjiagang, China), and informed consent was provided by the parents of the patients. Between August 2012 and February 2014, 80 pediatric patients ( $<3$ years old) hospitalized with pneumonia met the inclusion criteria and were enrolled in the present study. Among them were 42 males and 38 females, with a mean age of $1.2 \pm 0.9$ years. The patients were divided into two groups according to their diagnosis. In group 1, which comprised patients with simple pneumonia (24 males and 22 females; mean age, $1.2 \pm 0.9$ years), all patients presented with dyspnea, but the dyspnea was not accompanied by HF. Group 2 comprised patients with pneumonia accompanied by HF (18 males and 16 females; mean age, $1.1 \pm 0.8$ years). The diagnostic criteria for pneumonia and HF used in the present study were based on the Nelson Textbook of Pediatrics (11) (Figs. 1 and 2). In addition, 20 healthy children were enrolled in the study as negative controls. There were no statistical differences in the age and gender of the subjects among the three groups. All patients who participated in this study were confirmed to not suffer from any congenital heart disease or hepatic or renal disorders. The patients received treatments that were in accordance with the guidelines for pneumonia and/or HF. Briefly, for pneumonia, antibiotics were administered, and oxygen support, cough relief and sputum reduction were performed. For pneumonia accompanied by HF, alongside the previously mentioned treatments, conscious sedation, cardiotonic treatment, diuresis, and blood vessel expansion were performed.

Clinical specimens. Venous blood $(2 \mathrm{ml})$ was collected from all patients twice for plasma BNP detection. The first samples were collected on the day the patients were enrolled in the study, prior to treatment, and the second samples on the day the patients were discharged from the hospital, following the disappearance of the symptoms. The Triage ${ }^{\circledR}$ BNP automated immunoassay systems and reagents (Biosite Diagnostics, Inc., San Diego, CA, USA) were used to detect the plasma BNP levels, according to the manufacturer's instructions (12). During the evaluation, blood samples were collected in tubes, which contained potassium EDTA. A fluorescence immunoassay kit (Triage; Biosite Diagnostics, Inc.) was used for the quantitative determination of BNP in the plasma specimens. The analytic sensitivity, stability characteristics and precision of the system have been previously described $(13,14)$.

Statistical analysis. The data are presented as the mean \pm standard deviation. Analysis of variance and $\chi^{2}$ tests were performed in order to determine the statistical differences among the different groups. All P-values were determined by two-sided tests. $\mathrm{P}<0.05$ was considered to indicate a statistically significant difference. The statistical analysis of the data was performed using SPSS 10.0 software (SPSS Inc., Chicago, IL, USA).

\section{Results}

Plasma BNP levels in patients prior to treatment. The plasma BNP assays performed upon the enrollment of the patients in the
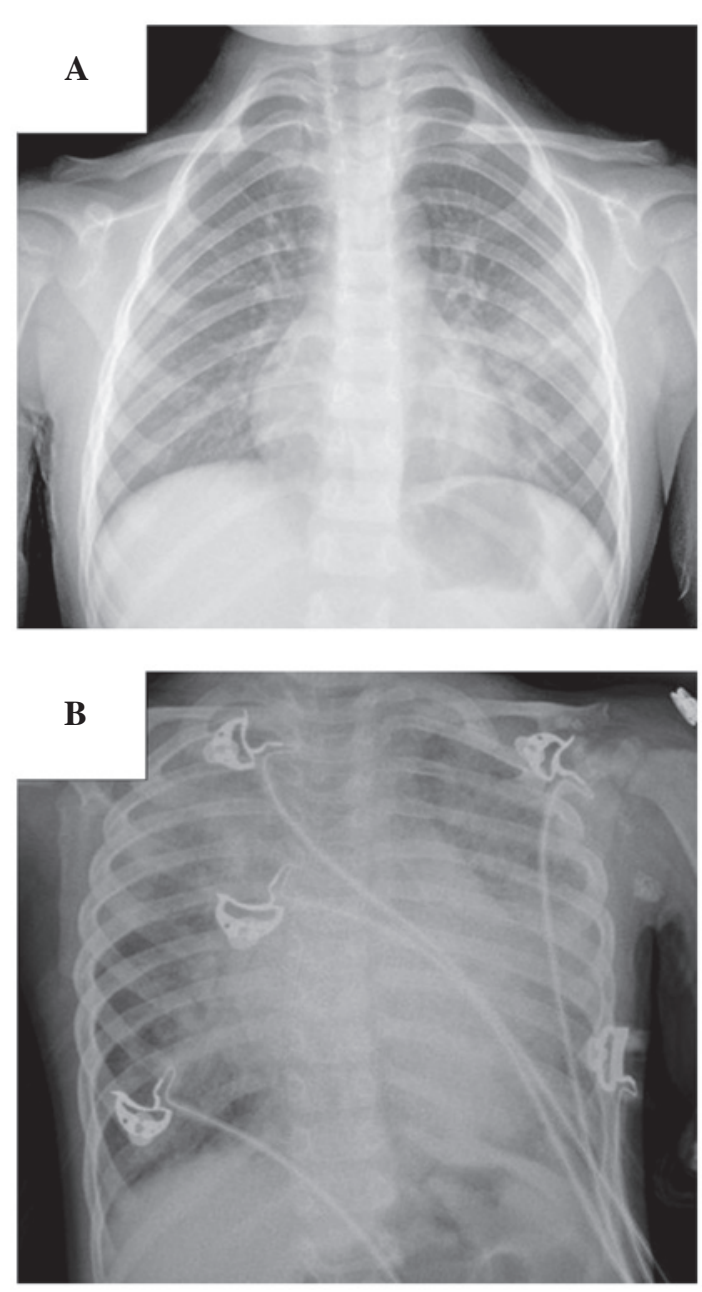

Figure 1. Chest radiograph of (A) patients with pneumonia: Diffuse distribution of spot or schistic shadow, localized large consolidation shadow in the lobes of the lung; (B) patients with pneumonia accompanied by heart failure: Diffuse exudation in pulmonary alveoli, diffuse schisitc shadow in both lungs, enlargement of podoid.

study showed that the BNP levels of group $2(750 \pm 120 \mathrm{pg} / \mathrm{ml})$ were significantly higher than those of group $1(135 \pm 50 \mathrm{pg} / \mathrm{ml})$ $(\mathrm{P}<0.05$, group 2 vs. group 1; Fig. 3); however, no significant difference was observed in the BNP levels between group 1 and the negative control $(125 \pm 34 \mathrm{pg} / \mathrm{ml})(\mathrm{P}>0.05)$.

Plasma BNP level in patients following treatment. The results of the plasma BNP assays performed upon the enrollment and prior to the discharge of the patients showed that, in group 2, the BNP levels of the patients $(750 \pm 120 \mathrm{pg} / \mathrm{ml})$ were significantly higher upon enrollment than those prior to discharge $(115 \pm 45 \mathrm{pg} / \mathrm{ml})(\mathrm{P}<0.05$, group 2 at enrollment vs. group 2 at discharge; Fig. 4). The BNP levels in group 1, however, showed no significant difference between the specimens collected upon enrollment $(135 \pm 50 \mathrm{pg} / \mathrm{ml})$ and those collected prior to discharge $(105 \pm 86 \mathrm{pg} / \mathrm{ml})(\mathrm{P}>0.05$, group 1 at enrollment vs. group 1 at discharge; Fig. 4).

\section{Discussion}

The discovery of ANP $>30$ years ago (15) was a milestone for the implementation of a laboratory marker of HF in the 

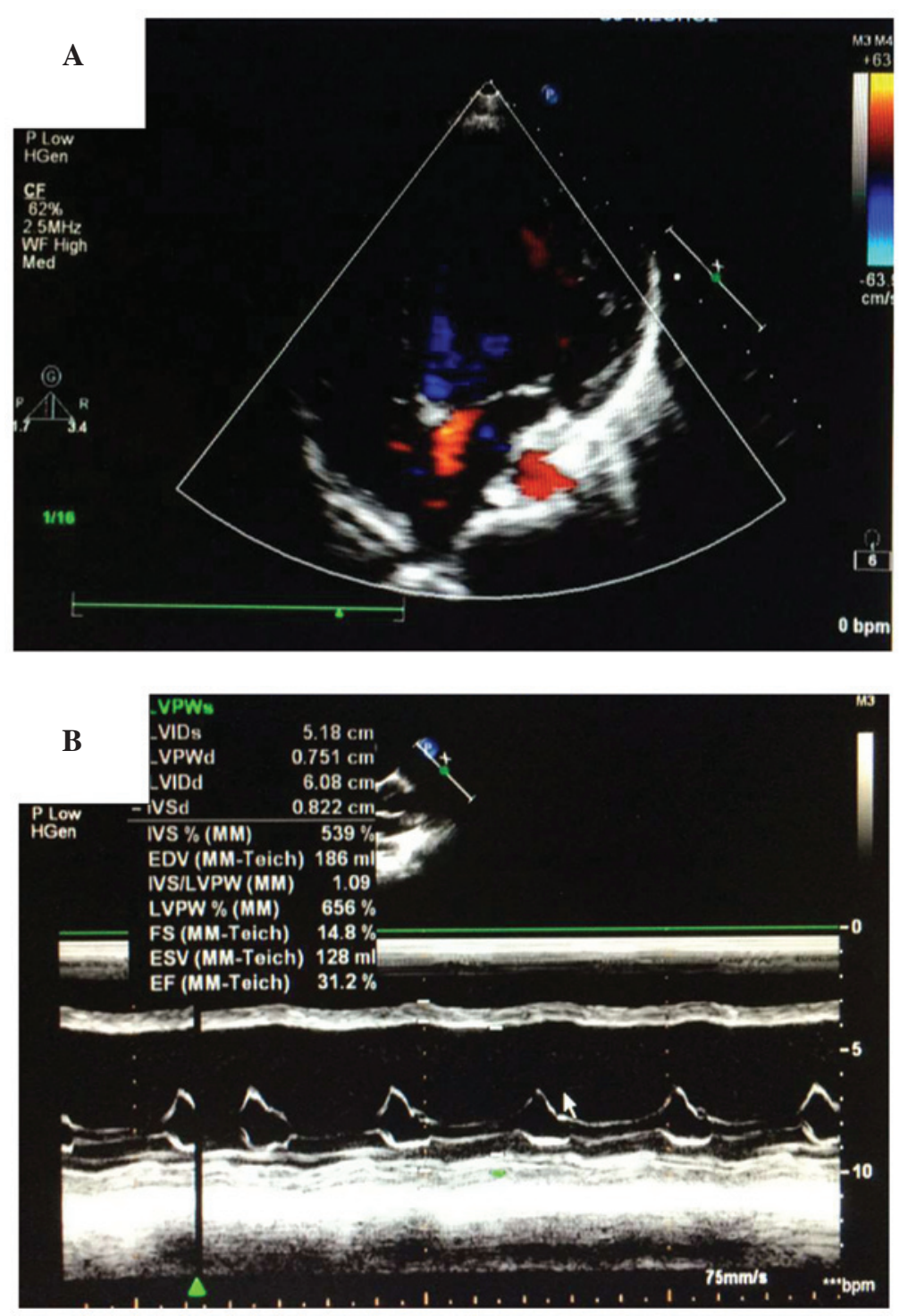

Figure 2. Ultrasonic cardiogram of patients with pneumonia accompanied by heart failure. (A) Color Doppler echocardiography shows left ventricular enlargement. (B) M-mode ultrasonic cardiogram shows that the ejection fraction was only $31.2 \%$.

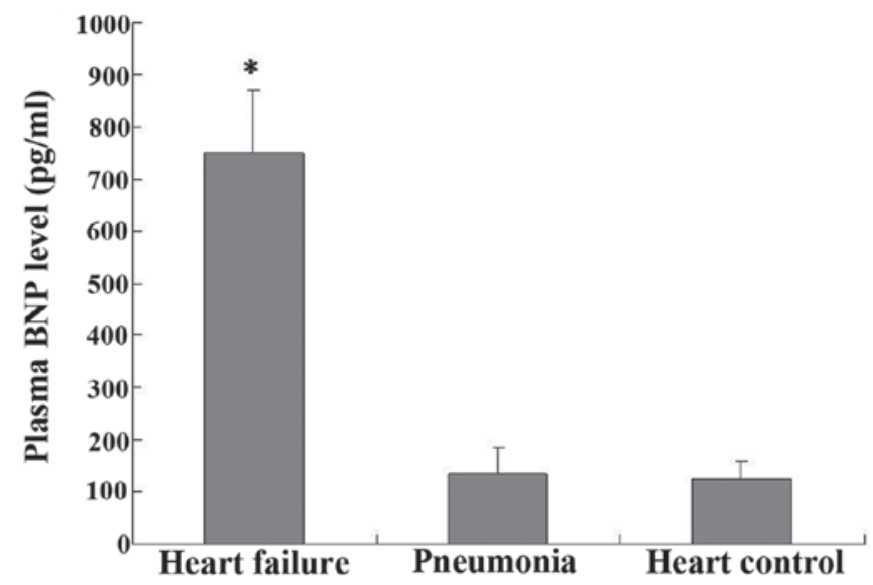

Figure 3. Plasma BNP assay performed on the day of the patients' enrollment showed that the BNP levels in the patients with pneumonia accompanied by heart failure were significantly higher than the BNP levels in the patients with simple pneumonia $\left({ }^{*} \mathrm{P}<0.05\right)$. No significant differences were observed in the BNP levels between the patients of the simple pneumonia group and those of the healthy control group. Data are presented as the mean \pm standard deviation. BNP, B-type natriuretic peptide.

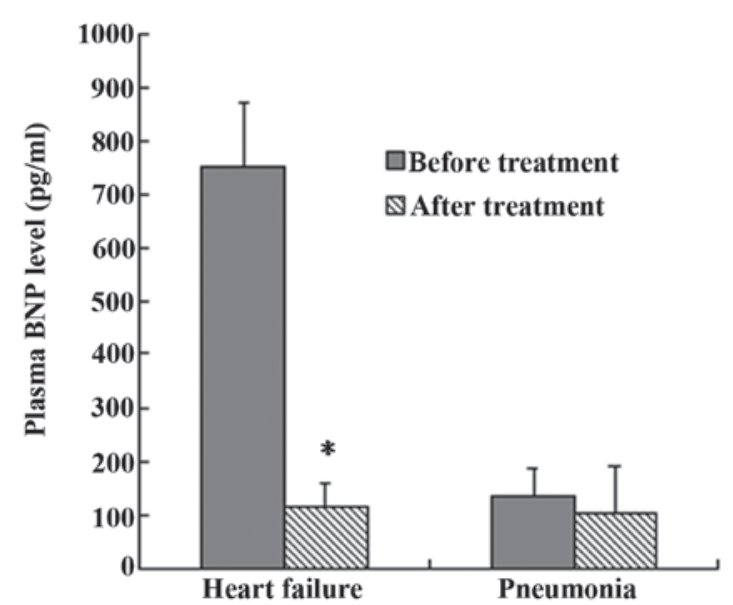

Figure 4. Plasma BNP assay on the day of the patients' enrollment and the day of discharge. In the group of patients with pneumonia accompanied by heart failure, the BNP levels upon enrollment were significantly higher than those prior to discharge $\left({ }^{*} \mathrm{P}<0.05\right)$, whereas no significant difference was observed between the BNP levels of the specimens from patients with simple pneumonia collected at the two time-points. Data are presented as the mean \pm standard deviation. BNP, B-type natriuretic peptide. 
clinical setting; soon thereafter, it was discovered that the heart also secretes BNP, a second NP (16). It is now apparent that the heart is also an endocrine gland releasing NPs from the hemodynamically stressed myocardium as a result of increased atrial or ventricular myocardial stretch or strain. BNP is expressed in the myocardium, brain and adrenal glands; however, the main source of BNP synthesis and secretion is the heart (1). The BNP system is considered to be one of the most important hormonal regulators of cardiovascular homeostasis and function $(1,2,4)$. Furthermore, it has been reported that this family of cardiovascular NPs plays a paracrine and autocrine role in the maintenance of the myocardial and vascular structure and function $(3,4)$. Soon after its discovery, the first finding of increased ANP plasma concentrations in patients with HF was reported (17). The potential for a plasma marker in HF was thereby raised, and investigations in this area have since received significant attention, with a particular focus on clinical applications. In subsequent comparative clinical studies, BNP emerged as a superior diagnostic marker compared with ANP-derived forms $(1,12)$. The significance of BNP in the diagnosis and risk stratification of patients with HF, as well as various other cardiac conditions, has also been largely investigated during the last two decades (12-14). Studies have focused on the impact of the NPs on disease and treatment monitoring, and their effect on patient management has been demonstrated $(1,2,4,12,13,18)$.

In clinical terms, HF is a syndrome with typical symptoms and signs, such as fatigue and breathlessness, and pulmonary crackles and elevated jugular venous pressure, respectively. Patients with HF may have either a reduced or preserved left ventricular ejection fraction. The diagnosis of HF can be complicated, since the clinical features of the condition are not always sensitive or specific, particularly in pediatric patients. The challenges of diagnosing HF in children emphasize the importance of evaluating whether other biomarkers can assist the diagnosis of the condition. Furthermore, the characteristics of these other biomarkers should be examined for their prognostic utility and usefulness in guiding therapy. The NPs, including BNP, have been shown to be useful in facilitating the diagnosis, prognosis, and management of $\mathrm{HF}$ in adults. BNP is secreted into the bloodstream by cardiac myocytes in response to increased ventricular wall stress, hypertrophy and volume overload, and its levels are increased in HF; therefore, BNP appears to be a promising marker for $\operatorname{HF}(5,6)$.

The present study focused on the differential diagnostic value of BNP in pneumonia accompanied by HF in pediatrics patients. The Biosite Triage BNP assay, which was the first device to receive the Food and Drug Administration approval in the United States, was used for the definitive diagnosis of congestive HF in patients within an acute setting with dyspnea (12). In the present study, the plasma BNP levels in patients with pneumonia, pneumonia accompanied by HF and healthy pediatric patients were detected, and it was found that the BNP levels in patients with pneumonia accompanied by HF were significantly higher than those in the patients with simple pneumonia. No differences were observed in the BNP levels between the patients with simple pneumonia and those in the healthy control group. The BNP levels of the patients were also compared prior to and following treatment. It was observed that, prior to treatment, the BNP levels in the patients with pneumonia accompanied by HF were significantly higher than those following treatment, which was not the case with the patients in the simple pneumonia group. No differences were observed in the BNP levels of the patients of the simple pneumonia group prior to treatment compared with those following treatment.

Previous studies have investigated the prognostic value of BNP in HF and consistent findings have shown that BNP is an independent predictor of all-cause and cardiovascular mortality, as well as other cardiac outcomes, such as worsening HF and hospitalization (19-22). In addition, these studies suggested that BNP levels that were measured prior to discharge or post-treatment were better predictors of prognosis than those measured at other time-points; however, the aforementioned studies focused mainly on adult patients. By contrast, the present study investigated the differential diagnostic value of BNP in pediatric patients with pneumonia accompanied by HF without congenital cardiac disease.

In conclusion, BNP may represent a useful biomarker that could be used for therapeutic monitoring and to help distinguish pediatric patients with pneumonia accompanied by HF from pediatric patients with simple pneumonia. BNP may therefore become a useful biomarker with diagnostic and prognostic value in pediatric patients with pneumonia accompanied by HF.

\section{Acknowledgements}

This study was supported by grants from the Science and Technology Research Development Project of Suzhou City, China (grant no. SYSD2012001).

\section{References}

1. Clerico A, Giannoni A, Vittorini S, et al: Thirty years of the heart as an endocrine organ: Physiological role and clinical utility of cardiac natriuretic hormones. Am J Physiol Heart Circ Physiol 301: H12-H20, 2011.

2. Cantinotti M, Giovannini S, Murzi B and Clerico A: Diagnostic, prognostic and therapeutic relevance of B-type natriuretic hormone and related peptides in children with congenital heart diseases. Clin Chem Lab Med 49: 567-580, 2011.

3. Hunt PJ, Richards AM, Nicholls MG, et al: Immunoreactive amino-terminal pro-brain natriuretic peptide (NT-PROBNP): A new marker of cardiac impairment. Clin Endocrinol (Oxf) 47: 287-296, 1997.

4. Mair J, Friedl W, Thomas S and Puschendorf B: Natriuretic peptides in assessment of left-ventricular dysfunction. Scand J Clin Lab Invest Suppl 230: 132-142, 1999.

5. McKelvie RS, Moe GW, Ezekowitz JA, et al: The 2012 Canadian Cardiovascular Society heart failure management guidelines update: Focus on acute and chronic heart failure. Can J Cardiol 29: 168-181, 2013.

6. McMurray JJ, Adamopoulos S, Anker SD, et al; ESC Committee for Practice Guidelines: ESC Guidelines for the diagnosis and treatment of acute and chronic heart failure 2012: The Task Force for the Diagnosis and Treatment of Acute and Chronic Heart Failure 2012 of the European Society of Cardiology. Developed in collaboration with the Heart Failure Association (HFA) of the ESC. Eur Heart J 33: 1787-1847, 2012.

7. Metra M, Ponikowski P, Dickstein K, et al: Advanced chronic heart failure: A position statement from the Study Group On Advanced Heart Failure of the Heart Failure Association of the European Society of Cardiology. Eur J Heart Fail 9: 684-694, 2007.

8. Heart Failure Society of America, Lindenfeld J, Albert NM, Boehmer JP, et al: HFSA 2010 comprehensive heart failure practice guideline. J Card Fail 16: e1-e194, 2010.

9. Felker GM, Adams KF Jr, Konstam MA, et al: The problem of decompensated heart failure: Nomenclature, classification and risk stratification. Am Heart J 145 (Suppl 2): 18-25, 2003. 
10. Arnold LM, Crouch MA, Carroll NV and Oinonen MJ: Outcomes associated with vasoactive therapy in patients with acute decompensated heart failure. Pharmacotherapy 26: 1078-1085, 2006

11. Kliegman RM, Stanton BMD, St. Geme J, Schor N and Behrman RE (eds): Part XIX, Respiratory System. In: Nelson Textbook of Pediatrics, 19th Edition. Saunders (Elsevier), Philadephia, PA, 2015.

12. Maisel A, Krishnaswamy P, Nowak RM, et al: Rapid measurement of B-type natriuretic peptide in the emergency diagnosis of heart failure. N Engl J Med 347: 161-167, 2002.

13. Morrison LK, Harrison A, Krishnaswamy P, et al: Utility of a rapid B-natriuretic peptide assay in differentiating congestive heart failure from lung disease in patients presenting with dyspnea. J Am Coll Cardiol 39: 202-209, 2002.

14. Mair J, Hammerer-Lercher A and Puschendorf B: The impact of cardiac natriuretic peptide determination on the diagnosis and management of heart failure. Clin Chem Lab Med 39: 571-588, 2001.

15. de Bold AJ, Borenstein HB, Veress AT, et al: A rapid and potent natriuretic response to intravenous injection of atrial myocardial extracts in rats. Life Sci 28: 89-94, 1981.

16. Saito $\mathrm{Y}$, Nakao $\mathrm{K}$, Itoh $\mathrm{H}$, et al: Brain natriuretic peptide is a novel cardiac hormone. Biochem Biophys Res Commun 158: 360-368, 1989

17. Burnett JC, Kao PC, Hu DC, et al: Atrial natriuretic peptide elevation in congestive heart failure in human. Science 231 $1145-1147,1986$
18. Frankenstein L, Nelles M, Slavutsky M, et al: Beta-blockers influence the short-term and long-term prognostic information of natriuretic peptides and catecholamines in chronic heart failure independent from specific agents. J Heart Lung Transplant 26: 1033-1039, 2007.

19. Di Angelantonio E, Chowdhury R, Sarwar N, et al: B-type natriuretic peptides and cardiovascular risk: Systematic review and meta-analysis of 40 prospective studies. Circulation 120: 2177-2187, 2009.

20. Doust JA, Pietrzak E, Dobson A and Glasziou P: How well does B-type natriuretic peptide predict death and cardiac events in patients with heart failure: systematic review. BMJ 330: 625, 2005.

21. Vesbianu D, Vesbianu C, Bernstein P, et al: Plasma brain natriuretic peptide-an independent predictor of mortality and rehospitalization in congestive heart failure-a meta-analysis. World Heart J 1: 349-354, 2008.

22. Oremus M, Raina PS, Santaguida P, et al: A systematic review of $\mathrm{BNP}$ as a predictor of prognosis in persons with coronary artery disease. Clin Biochem 41: 260-265, 2008. 